\title{
Microcrystalline parameters in native and acid-modified starches
}

\author{
H SOMASHEKARAPPA, R SOMASHEKAR*, VASUDEVA SINGH ${ }^{\dagger}$ and S Z ALI \\ Department of Studies in Physics, University of Mysore, Manasagangotri, Mysore 570 006, India \\ ${ }^{\dagger}$ Grain Science and Technology, Central Food Technological and Research Institute (CFTRI), Mysore 570 005, India
}

MS received 16 November 1998; revised 20 February 1999

\begin{abstract}
Crystallite size and lattice distortion parameters of starches from cereals, millet, pulse, root and tuber before and after acid modification were determined by wide angle X-ray scattering (WAXS) studies. The $(h k l)$ planes and cell parameters were obtained using a minimization program. Acid modification results in minor changes in $d$-spacing but there is considerable change in microcrystalline parameters, which has been reported here. Using these data, we have also estimated the changes in the mean shape of the coherent domains in terms of the shape ellipsoid in these starches for various types of acids treatment.
\end{abstract}

Keywords. X-rays; starch granules; acid modification; shape ellipsoid.

\section{Introduction}

Starch has been regarded as a semi-crystalline material since the classic work of Katz (1937) using X-ray diffraction technique. There are three different types of crystalline structures for starch granules which have been designated as A-, B- and C-types (Zobel 1964; French 1984). Transmission electron microscopic studies (Whistler and Turner 1955; Hizkuri and Nikuni 1957; Buttrose 1963) have provided parallel and confirming data on the nature of electron dense regions in starches. The microgranular structure has been observed to be accentuated by enzymic and acidic erosion (Nikuni 1978; Chabott et al 1978). Sarko and $\mathrm{Wu}(1978)$ have studied crystal structures of A-, B- and C-polymorphs of amylose by combination of $\mathrm{X}$-ray diffraction and computer-based structure refinement. Nara et al (1978) have reported that potato starch diffraction pattern becomes sharp with increase of sorption moisture and in areas of crystallinity were proportional to the amount of moisture. These methods do not consider the possible lattice distortions like paracrystalline disorder present in these starches. By deconvoluting the experimental profiles for instrumental broadening correction as suggested by Stokes (1948), Warren and Averbach (1950) have shown that these corrected Fourier coefficients can be used to separate out crystal size and lattice distortion parameters. Somashekar et al (1989) and Hall and Somashekar (1991) have 'suggested a suitable single-order method to obtain these parameters fairly accurately. This method has been used to obtain crystallite size and lattice distortion parameters in the case of different native starch granules using

\footnotetext{
*Author for correspondence
}

photographic method (Vasudev Singh et al 1995). In continuation of these studies, we have examined the changes in microcrystalline parameters in these starches with acid modification of native starch granules.

\section{Materials}

Maize and tapioca starches were procured from commercial sources. Potato starch was a gift from W Kempf, Federal Research Centre for Cereal and Potato Processing, Detmold, Germany. Green gram pulse was procured from the local market. All chemicals used were of analytical grade. Starches from all other source materials were procured from local market and isolated in the laboratory as reported earlier (Singh and Ali 1987). The starches were acid-modified (33\% starch slurry) using $0.5 \mathrm{~N}\left(\mathrm{HCl}, \mathrm{HNO}_{3}, \mathrm{H}_{2} \mathrm{SO}_{4}\right.$ and $\left.\mathrm{H}_{3} \mathrm{PO}_{4}\right)$ for $1.5 \mathrm{~h}$ at $50^{\circ} \mathrm{C}$ with occasional stirring. At the end they were neutralized with $\mathrm{NaOH}$ and washed till they were free from respective anions. The starches were dried and ground using Fritsch pulverizer with $2.0 \mathrm{~mm}$ screen. Samples had a moisture content of about $12.5 \%$ and protein content between $0.1 \%$ and $0.3 \%$.

\section{Methods}

\subsection{Recording of X-ray diffraction pattern}

$\mathrm{X}$-ray diffraction pattern recorded using a Philips X-ray diffractometer system PW 1710 equipped with a monochromator with $\mathrm{Cu}$ target $(\lambda=1.5402 \AA)$. The conditions for recording are the following: $35 \mathrm{kV}, 15 \mathrm{~mA}$, scan speed of $2.4 \%$ min and scanning angle $(2 \theta)$ from $5-30^{\circ}$. Figure 1 shows the X-ray patterns obtained for potato, tapioca, 
green gram, finger millet, wheat and maize starches before and after acid modification. Here we have carried out profile analysis for the reflections which are well separated.

\subsection{Analysis of $X$-ray diffraction pattern}

$\mathrm{X}$-ray reflections obtained for native and acid-modified starches were identified by a computer structural refinement program wherein a multidimensional minimization method (De Wolff 1968; Press et al 1988) has been used. For minimization, 7-8 observed reflections were used. The cell parameters obtained for different starches are given in table 1 along with the volume of the unit cell. It should be mentioned here that we could not fit all the observed reflections using the cell parameters reported earlier (Sarko and Wu 1978). With acid modification there is no significant change in the cell parameters and hence in the volume of the unit cell.

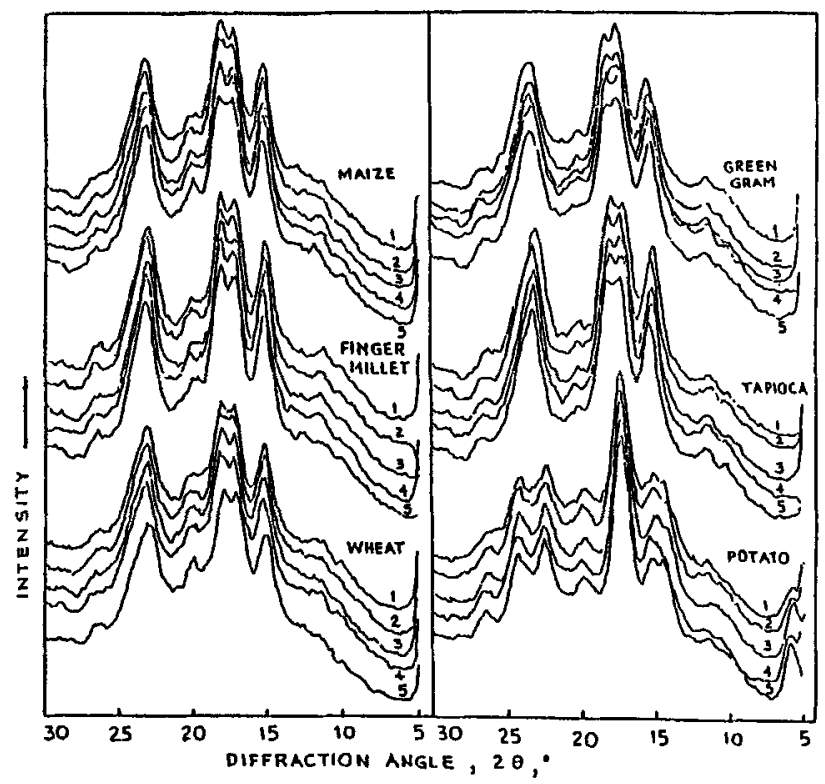

Figure 1. X-ray diffractograms of various starches before and after modification with different acids. 1. native, 2-5: modified with $\mathrm{HCl}, \mathrm{HNO}_{3}, \mathrm{H}_{2} \mathrm{SO}_{4}$ and $\mathrm{H}_{3} \mathrm{PO}_{4}$ respectively $\left(0.5 \mathrm{~N}, 50^{\circ} \mathrm{C}\right.$, $1.5 \mathrm{~h})$.

\subsection{Profile analysis using Fourier method}

The intensity profile of the X-ray reflection from starch granules is a function of distribution of crystal sizes $\langle D\rangle$ $\left(=N d_{(h k l)}\right)$ and of the lattice distortion. These are related through the Fourier coefficients $A(n)$ of the profile intensity $I(s)$ by

$$
I(s)=\sum_{n=-\infty}^{\infty} A(n) \cos \left\{2 \pi n d\left(s-s_{0}\right)\right\}
$$

here $s=s_{0}$ is the value of $s=(\sin \theta / \lambda)$ at the peak of the profile, $d$ the mean spacing of the lattice plane causing the reflection and $n$ the harmonic number.

Also the Fourier coefficient can be factorized into size $A_{s}(n)$ and disorder coefficients $A_{d}(n)$;

$$
A(n)=A_{s}(n) \cdot A_{d}(n),
$$

and these are not normalized. The reasons for using nonnormalized Fourier coefficients in all these calculations are (i) truncation of the profile and (ii) error in the background estimation and these affect the low-order Fourier coefficients of the intensity profile as explained in detail by Somashekar et al (1989). Under these circumstances, to use higher order coefficients, various analytical forms for the distribution of crystallite sizes have been investigated by Hall and Somashekar (1991) and it has been reported that an asymmetric function for the crystal size distribution gives fairly good results. Using this exponential function, Hall and Somashekar arrive at the following expression

$$
\begin{aligned}
& A_{s}(n)=A(0)(1-n /\langle N\rangle) ; \quad n \leq p, \\
& A_{d}(n)=A(0)[\exp \{-\alpha(n-p)\} / \alpha\langle N\rangle] ; \quad n \geq p .
\end{aligned}
$$

Here $p$ is the smallest crystal size. The crystal size is given by

$$
D=\left\langle N>d_{(h k l)},\right.
$$

where $A_{d}(n)$ is the disorder coefficient for a paracrystal with separation of neighbouring lattice planes having a Gaussian distribution of standard deviation $\sigma$ given by

$$
A_{d}(n)=\exp \left(-2 \pi^{2} m^{2} n g^{2}\right)
$$

Table 1. Cell parameters for different starch granules.

\begin{tabular}{lccccccc}
\hline Sample & \multicolumn{1}{c}{$\mathrm{a}(\AA)$} & $\mathrm{b}(\AA)$ & $\mathrm{c}(\AA)$ & $\alpha(\mathrm{deg})$ & $\beta(\mathrm{deg})$ & $\gamma(\mathrm{deg})$ & Volume $\left(\AA^{3}\right)$ \\
\hline Potato & $19.09 \pm 0.02$ & $15.33 \pm 0.03$ & $6.12 \pm 0.02$ & 90 & 90 & 90 & 1790.63 \\
Tapioca & $15.32 \pm 0.01$ & $13.75 \pm 0.01$ & $6.43 \pm 0.01$ & 90 & 90 & 90 & 1353.97 \\
Green gram & $\mathbf{8 . 6 5} \pm 0.01$ & $8.65 \pm 0.01$ & $17.76 \pm 0.02$ & 90 & 90 & 90 & 1327.81 \\
Finger millet & $15.55 \pm 0.04$ & $8.79 \pm 0.01$ & $6.90 \pm 0.01$ & 90 & 90 & 90 & 943.15 \\
Maize & $15.57 \pm 0.02$ & $15.57 \pm 0.02$ & $5.83 \pm 0.01$ & 90 & 90 & 90 & 1414.22 \\
Wheat & $17.44 \pm 0.03$ & $17.44 \pm 0.03$ & $5.11 \pm 0.01$ & 90 & 90 & 90 & 1553.87 \\
\hline
\end{tabular}




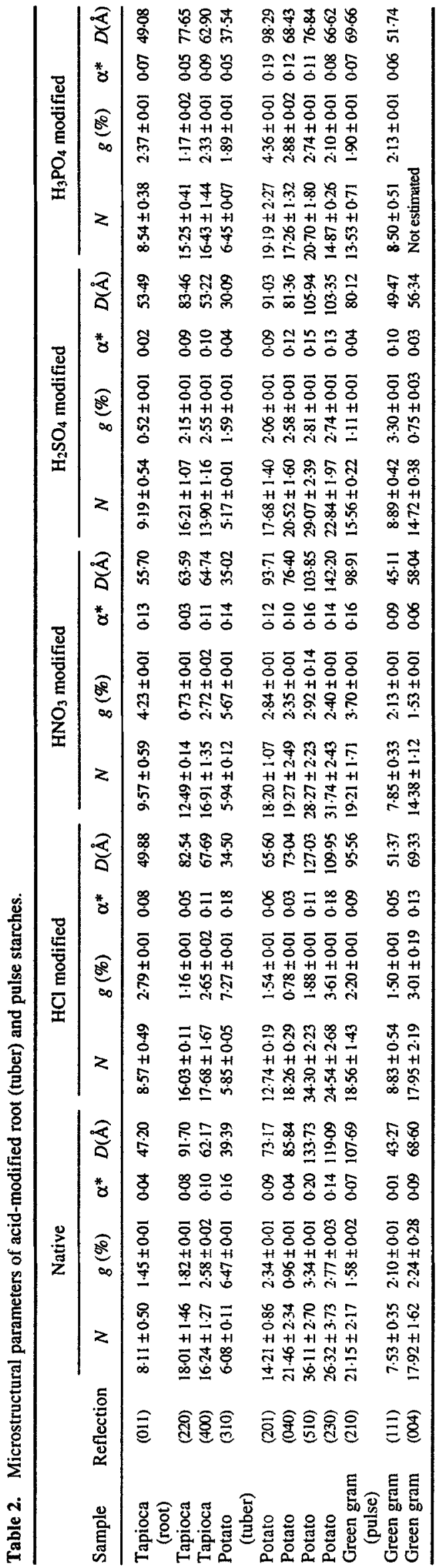

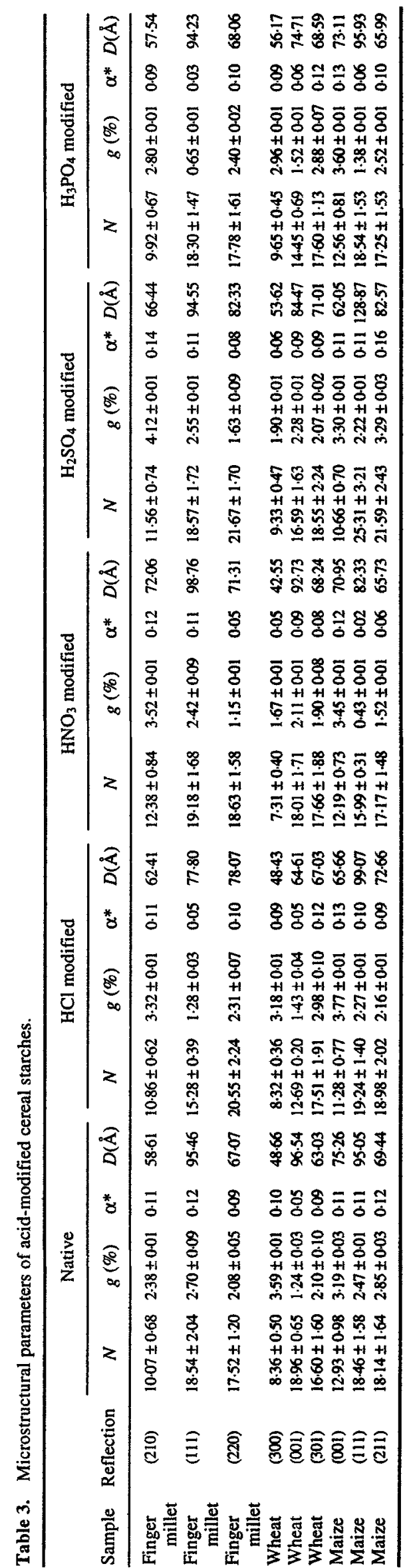




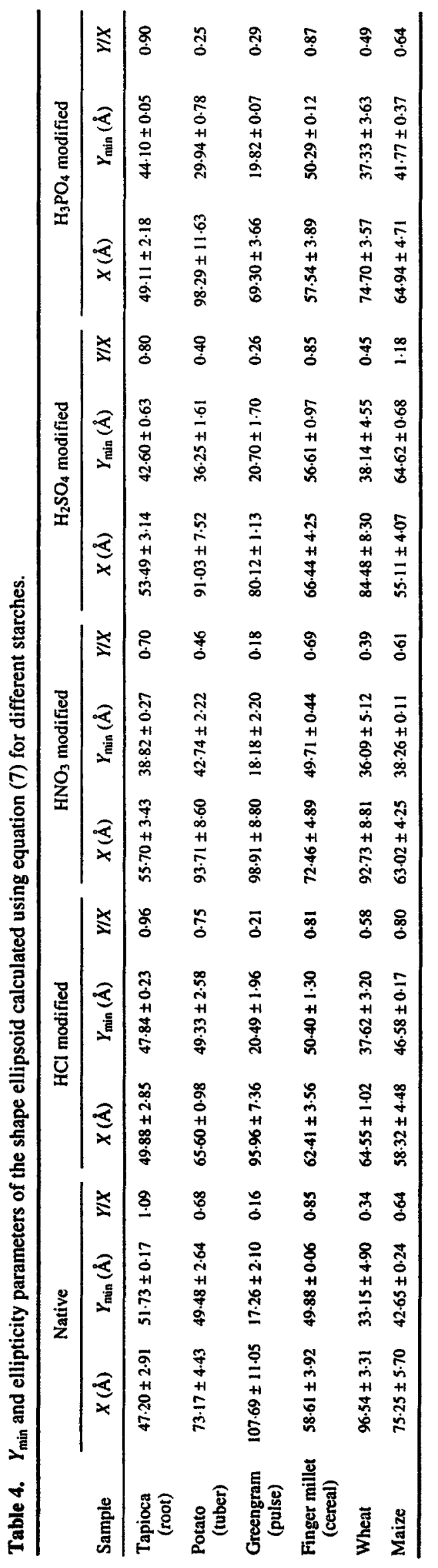


where $m$ is the order of reflection and $g$ the paracrystallinity of lattice distortion parameter. Using (1), (2), (3) and (5) along with the experimental intensity profile data, it is possible to obtain the crystal size and lattice distortion parameters.

The experimental profiles were corrected for Lorentz and polarization factors. Initial values of $g$ and $\langle N\rangle$ were obtained using the method of Nandi et al (1984). Using these values in the above mentioned equations the corresponding values for the width of the distribution function are obtained. These are only rough estimates, but the refinement procedure must be sufficiently robust to start with such inaccurate values. Here we compute

$$
\Delta^{2}=\left[I_{\text {cal }}-\left(I_{\text {exp }}+\text { BG }\right)\right]^{2} / \text { number of points. }
$$

The value of $\Delta$ was divided by half the maximum value of intensity so that it is expressed relative to the mean value of intensities and this function is minimized. For refinement, the multidimensional minimization algorithm of SIMPLEX method was used. Here BG refers to inaccuracy in background estimation and this was less than $0.1 \%$ of the normalized peak intensity. Here we have used an asymmetric exponential function for the crystal size distribution as this gives better results. It was observed that the variation of $\langle N\rangle, p$ and $\alpha$ defined in (6) with respect to $g$ for exponential distribution function are almost constant and under these circumstances, the average values of parameters $\langle N\rangle, p$ and $\alpha$ were used to determine the $g$ value. All the necessary computer programs were written in FTN77 language and compiled and executed using IBM Pentium $166 \mathrm{MHz}$ machine.

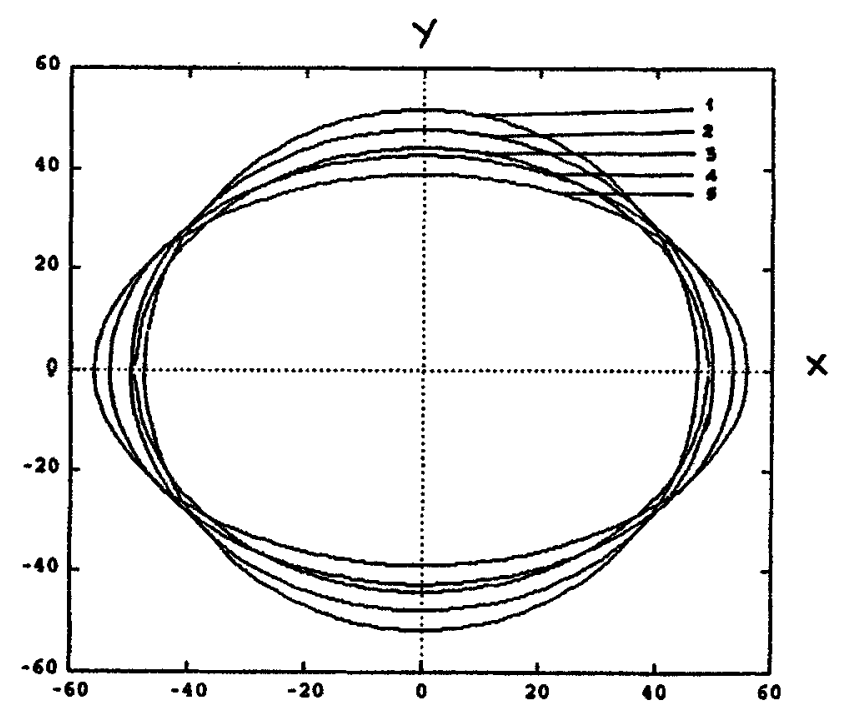

Figure 2. Variation of shape ellipsoid for tapioca starch with acid modification. 1. native, modified with $2 . \mathrm{HCl}, 3 . \mathrm{H}_{3} \mathrm{PO}_{4}, 4$. $\mathrm{H}_{2} \mathrm{SO}_{4}, 5 . \mathrm{HNO}_{3}$.

\section{Results and discussion}

In all cases, the goodness of the fit, between the experimental and simulated profile was less than $3 \%$ of the mean value calculated using (6). The profiles were recalculated using microstructural parameters given in tables 2 and 3 . Tables 2 and 3 give various parameters like the number of unit cells $\langle N\rangle$, crystal size $D$ in $\AA$, lattice distortion $g$ in $\%$ and enthalpy $\alpha^{*}$ which defines equilibrium state of microcrystals in starch of various roots, pulses and cereals (Hindeleh and Hosemann 1991) (native and acid-modified).

From tables $1-3$, it is rather difficult to arrive at any definite conclusions. In order to put these results in a better perspective, we have used these data (i.e. tables 13) for computing the mean shape of the coherent domains (crystallites) in terms of the shape ellipsoid (Hosemann and Bagchi 1962).

Taking one of the $D_{h k l}^{\prime}$ s to be along $X$-axis, we have fitted all other values of each sample before and after acid treatment into an ellipsoid with principal axes $X$ and $Y$ using the equation

$$
\left(2 / D_{h k l}\right)^{2}=(\cos \phi / Y)^{2}+(\sin \phi / X)^{2},
$$

where $\phi$ is the angle between $(h k l)$ planes. This $\phi$ can be determined using the data given in table 1. By iteration procedure, $Y_{\min }$ was computed using the data for all $(h k l)$ planes in a given sample and hence the ellipticity $Y / X$ was obtained. The data given in table 4 indicate that acid treatment do modify the mean shape of the coherent crystallites described in terms of the ellipticity. Figure 2 shows the shape of the ellipsoid in $X Y$ plane with $D_{011}$ along $X$-axis and observed changes with acid treatment. It is to be emphasized here that $Y_{\min }$ estimated value depends on the number of reflections used.

The smallest shape ellipsoid was observed in tapioca, finger millet and maize with $\mathrm{HNO}_{3}$ modified starches. $\mathrm{HCl}$-modified starches like potato and wheat show significant increase in ellipticity indicating more ordering in these starches. The averaged $\alpha^{*}$, the enthalpy value, remains almost constant with acid modification of starches indicating that phase stabilization is not affected with acid treatment.

\section{Conclusion}

Although there is not much change in cell parameters of starches after acid modification, there are significant changes in the shape of the crystallite domains described here in terms of the ellipticity, these changes indicate the degradation in starch granules due to acid modification which may help in understanding the digestion aspect of these materials. The phase stabilization is not affected with acid treatment which is indicated by the value of $\alpha^{*}$, the enthalpy. 


\section{Acknowledgements}

One of us (HS) thanks the University of Mysore, Mysore, for a Teacher Fellowship and the authors thank Prof. Nagappa for computer facilities.

\section{References}

Buttrose M S 1963 Naturwissenschaften 12450

Chabott J F, Allen J E and Hood L F 1978 J. Food Sci. 43727

De Wolff P M 1968 J. Appl. Crystallogr. 1108

French D 1984 in Starch chemistry and technology (eds) R L Whistler, J N Bemiller and E F Paschall (New York: Academic Press) 2nd edn, p. 183

Hall I H and Somashekar R 1991 J. Appl. Crystallogr. 241051 Hindeleh A M and Hosemann R 1991 J. Mater. Sci. 265127 Hizukuri $S$ and Nikuni Z 1957 Nature (London) 180436

Hosemann R and Bagchi S N 1962 Direct analysis of diffraction by matter (Amsterdam: North Holland)
Katz J R 1937 Recl. Trav. Chim. Pays-Bas Beig. 56785

Nandi R K, Kuo H R, Schlosberg M, Wisseler G, Cohen J B and Crist B J 1984 J. Appl. Crystallogr. 1722

Nara Sh., Mori A and Komiya T 1978 Starch/Staerke 4111

Nikuni Z 1978 Starch/Staerke 30105

Press W, Flannery B P, Teukolsky S and Vettering W T 1988 Numerical recipes (Cambridge: University Press) p 284

Sarko A and Wu C H 1978 Starch/Staerke 3073

Singh V and Ali S Z 1987 Starch/Staerke 3973

Somashekar R, Hall I H and Carr P D $1989 \mathrm{~J}$. Appl. Crystallogr. 22363

Stokes A R 1948 Proc. Phys. Soc. London 61382

Vasudev Singh, Gopal Krishna Urs, Somashekarappa H, Ali S Z and Somashekar R 1995 Bull. Mater. Sci. 18549

Warren B E and Averbach B L 1950 J. Appl. Phys. 21595

Whistler R L and Turner E S 1955 J. Polym. Sci. 18153

Zobel H F 1964 in Methods in carbohydrate chemistry (eds) R L Whistler, R J Smith and J N Bemiller (New York: Academic Press) 